\title{
Topological phase transition in quantum-heat-engine cycles
}

\author{
Mojde Fadaie, ${ }^{*}$ Elif Yunt, ${ }^{\dagger}$ and Özgür E. Müstecaplıoğlu ${ }^{\ddagger}$ \\ Department of Physics, Koç University, 34450 Sariyer, Istanbul, Turkey
}

(Received 5 July 2018; published 20 November 2018)

\begin{abstract}
We explore the signatures of a topological phase transition (TPT) in the work and efficiency of a quantum heat engine, which uses a single-layer topological insulator, stanene, in an external electric field as a working substance. The magnitude of the electric field controls the trivial and topological insulator phases of the stanene. The effect of the TPT is investigated in two types of thermodynamic cycles, with and without adiabatic stages. We examine a quantum Otto cycle for the adiabatic case and an idealized Stirling cycle for the nonadiabatic case. In both cycles, investigations are done for high and low temperatures. It is found that the Otto cycle can distinguish the critical point of the TPT as an extremum point in the work output with respect to applied fields at all temperatures. The Stirling cycle can identify the critical point of the TPT as the maximum work point with respect to the applied fields only at relatively lower temperatures. As temperatures increase toward room temperature, the maximum work point of the Stirling cycle shifts away from the critical point of the TPT. In both cycles, increasing the temperature causes considerable enhancement in work and efficiency from the order of $\mathrm{meV}$ to $\mathrm{eV}$.
\end{abstract}

DOI: 10.1103/PhysRevE.98.052124

\section{INTRODUCTION}

Heat engines are one of the practical motivations and significant outcomes of the field of thermodynamics, and they had a high socioeconomic impact in the industrial revolution [1]. Along with the social and industrial developments, the list of working substances used in heat engines has been growing since the steam engine in the 18th century. The list includes three-level masers [2], cavity photons [3], spin systems [4], single atoms [5], atomic clusters [6], optomechanical systems [7], superconducting resonators [8], Dirac particles [9,10], graphene flakes [11-13], black holes [14], and ultracold atoms [15]. Heat engines that use working substances requiring quantum-mechanical descriptions are called quantum heat engines (QHEs). Their cycles [16] are studied under a quantum thermodynamical framework, which is an emerging and rapidly progressing field of research [16-20]. Experimental demonstrations of a single-atom heat engine [5] and QHEs with nitrogen-vacancy centers in diamond [21] and with cold $\mathrm{Rb}$ atoms [22] have been shown.

Typically, the working system of the engine remains in a single phase during a thermodynamic cycle. In practice, however, phase changes can happen during the engine operation and can enhance the efficiency. Power plants, for example, can be modeled by the Rankine cycle, which is based upon a water-steam phase transition. Recently, it has been argued that diverging fluctuations at a second-order phase transition can help to achieve Carnot efficiency in a quantum Otto engine [23]. More recently, it has been shown that a quantum phase transition (QPT) of an interacting spin working system

\footnotetext{
*mfadaei@ku.edu.tr

†eyunt@ku.edu.tr

‡omustecap@ku.edu.tr
}

enables Carnot efficiency to be reached [24]. Inspired by these results, we ask whether a topological phase transition (TPT) during a quantum engine cycle can have any significant effects on the efficiency. Furthermore, we explore whether such effects can be used to probe a TPT. In contrast to ordinary QPTs, TPTs have no local order parameter associated with symmetry breaking. A TPT is described by a bulk invariant (Chern number), which is an integer that changes to another integer at the TPT. In the case of a gap closing at the TPT, we expect that the global nature of the work could capture the TPT qualitatively in certain cycles.

Usually, statistical work distribution measurements are studied in suddenly and infinitesimally quenched systems to examine phase transitions in quantum critical models [25]. Quench protocols bring the systems out of equilibrium, and the work output is given by a probability distribution whose characteristic function can be related to the Loschmidt echo that can be determined in principle experimentally. It is shown that local quenches lead to edge singularities in the work distribution in a quantum critical system [26]. Our approach, on the other hand, is based upon a cyclic variation of a control parameter, instead of quenching, and it entails looking for signatures of the TPT in the work output of the cycle.

We emphasize that probing the TPT using the work efficiency of QHEs is possible for those two-dimensional (2D) materials, such as stanene, germanane, and silicene, for which it is established that bulk gap closing (under electric-field variation, for example) is associated with a topological phase transition $[27,28]$. We consider specifically a 2D monolayer stanene ( $\mathrm{Sn})$ as our working substance. Stanene is a counterpart of graphene for tin atoms [28-30] with low-buckled honeycomb geometry [31]. In contrast to graphene, it has a larger spin-orbit coupling [32,33], it can host the quantum spin Hall effect at room temperature for dissipationless electric currents [29], and its band structure, particularly the Dirac 
cone band gap, can be controlled with an out-of-plane electric field such that it exhibits a TPT between two-dimensional trivial and topological insulator (2DTI) phases depending on the applied electric field [27,34-36]. 2D Sn has been fabricated recently by molecular beam epitaxy [37]. Studies of the thermal properties of $\mathrm{Sn}$ have so far been limited to thermoelectrics [38]. Its outstanding properties, on the other hand, make it also an ideal candidate for a working substance of a room-temperature topological QHE. We note that using heat, instead of work, has been considered recently to detect dynamical phase transitions and Majorana modes [39]. It is proposed there that Floquet-Majorona phases can be used for QHEs or heat pumps. There are also theoretical investigations of TPTs from the perspectives of Hill thermodynamics [40,41] and the Uhlmann phase [42,43]. Our simple model here can be envisioned as the first step toward QHEs with more sophisticated topological materials with TPTs.

By using an external electric field as the control parameter, we consider adiabatic (specifically the Otto cycle) and nonadiabatic (specifically a Stirling-type cycle as in Ref. [24]) thermodynamic cycles in which $\mathrm{Sn}$ undergoes a topological phase transition. We calculate the work output and efficiency of the cycles, and we determine and compare the signatures of the TPT in both types of cycles. We discover that the answer to our question for both cycles is positive, i.e., the TPT of Sn can be probed by using work and efficiency of QHE cycles. Highly distinct characteristic behaviors are obtained below and above the critical point of the TPT. Another advantage of the Sn working substance is that the topological QHE can operate around room temperature without the need for large external electric fields. At the end of this paper, we briefly discuss how to implement the cycle in an experimental setting using a graphene bilayer as a scaffold.

This paper is organized as follows: We review the theory and TPT of Sn in Sec. II. Results and discussions are presented in Sec. III in two subsections: the quantum Otto cycle case is presented in Sec. III A, and the nonadiabatic cycle case is presented in Sec. III B. In Sec. IV, a possible experimental realization is discussed. We conclude in Sec. V. The details of the calculations of the work output for both of the quantum thermodynamic cycles are given in Appendixes A and B.

\section{WORKING SUBSTANCE}

This section is a brief review of relevant properties of $\mathrm{Sn}$ for our QHE and TPT discussions. We consider a single layer of two-dimensional $\mathrm{Sn}$ in the $x y$ plane as the working substance for a QHE. An external electric field $\varepsilon_{z}$ is applied in the $z$ direction, perpendicular to the atomic layer. The system is described by a second-nearest-neighbor tight-binding model given in Ref. [28],

$$
\begin{aligned}
H= & -t \sum_{\langle i, j\rangle \alpha} c_{i \alpha}^{\dagger} c_{j \alpha}+i \frac{\lambda_{\mathrm{SO}}}{3 \sqrt{3}} \sum_{\langle\langle i, j\rangle\rangle \alpha \beta} v_{\mathrm{ij}} c_{i \alpha}^{\dagger} \sigma_{\alpha \beta}^{z} c_{j \beta} \\
& -i \frac{2 \lambda_{\mathrm{R}}}{3} \sum_{\langle\langle i, j\rangle\rangle \alpha \beta} \mu_{\mathrm{ij}} c_{i \alpha}^{\dagger}\left(\vec{\sigma} \times \overrightarrow{d_{i j}^{0}}\right)_{\alpha \beta}^{z} c_{j \beta} \\
& +l \sum_{i \alpha} \zeta_{i} \varepsilon_{z}^{i} c_{i \alpha}^{\dagger} c_{i \alpha} .
\end{aligned}
$$

The first term is the nearest-neighbor hopping term, and $t$ is the transfer energy. The sum is taken over all pairs $\langle i, j\rangle$ of the nearest-neighboring sites, and $c_{i \alpha}^{\dagger}$ and $c_{j \alpha}$ create and annihilate an electron with spin polarization $\alpha$ at site $i$, respectively. The second and third terms are the effective and intrinsic Rashba spin-orbit interactions with the corresponding coefficients $\lambda_{\text {SO }}$ and $\lambda_{\mathrm{R}}$, respectively. $\mu_{\mathrm{ij}}$ and $\zeta_{i}$ are equal to \pm 1 for two sublattices of Sn. $\vec{\sigma}$ denote the Pauli matrices. The coefficients $v_{\mathrm{ij}}$ are defined as

$$
v_{\mathrm{ij}}=\vec{d}_{i} \times \vec{d}_{j} /\left|\vec{d}_{i} \times \vec{d}_{j}\right|
$$

where $\vec{d}_{i}$ and $\vec{d}_{i}$ are the two nearest bonds connecting the next-nearest neighbors $\vec{d}_{i j}$ and $\vec{d}_{i j}^{0}=\vec{d}_{i j} /\left|\vec{d}_{i j}\right| . l$ is half of the perpendicular distance between two sublattices. The sum is taken over all pairs $\langle\langle i, j\rangle\rangle$ of the next-nearest neighbors. We note that the model describes other two-dimensional honeycomb structures as well $[28,44]$.

The low-energy effective Hamiltonian for stanene is derived from Eq. (1) around the $K_{\eta}$ point in Refs. [28,32] as

$$
H_{\eta}^{s}=\hbar v_{f}\left(\tau_{x} k_{x}-\eta k_{y} \tau_{y}\right)-\eta \lambda_{\mathrm{SO}} \tau_{z} s_{z}+l \varepsilon_{z} \tau_{z},
$$

where $\eta= \pm 1$ is for the $K$ and $K^{\prime}$ point, $v_{f}$ is the Fermi velocity, and $\tau_{x, y, z}$ are the Pauli matrices of the sublattice. $s_{z}$ is the third Pauli matrix depicting the spin in the $z$ direction. Here, we ignore the Rashba term in Ref. [28], as we will consider the behavior of the bands exactly at the Dirac points where the Rashba term vanishes. The energy spectrum of Eq. (3) is found to be [28]

$$
E_{\eta}^{s}(k)= \pm \sqrt{\hbar^{2} v_{f}^{2} k^{2}+\left(l \varepsilon_{z}-\eta \lambda_{\mathrm{SO}}\right)^{2}}
$$

where $k=\sqrt{k_{x}^{2}+k_{y}^{2}}$. There are in total four distinct energy eigenvalues, which are each twofold-degenerate.

The low-energy band structure of $\mathrm{Sn}$ in the vicinity of the $K$ point given by Eq. (4) shows that the energy gap $\Delta=2\left|\lambda_{\text {so }}-l \varepsilon_{z}\right|$ at $k=0$ is finite at $\varepsilon_{z}=0$; it decreases and closes at a critical value $\varepsilon_{\mathrm{cr}}$. The phase diagram of $\mathrm{Sn}$ with respect to the external electric field is shown in Fig. 1. The gap closing occurs at $\varepsilon_{z}= \pm \varepsilon_{c r}$. It is found that the gap closing is associated with the TPT such that for $\left|\varepsilon_{z}\right|<\varepsilon_{\mathrm{cr}} \mathrm{Sn}$ is a TI and for $\left|\varepsilon_{z}\right|>\varepsilon_{\mathrm{cr}}$ it is a band insulator $[27,28,36]$.

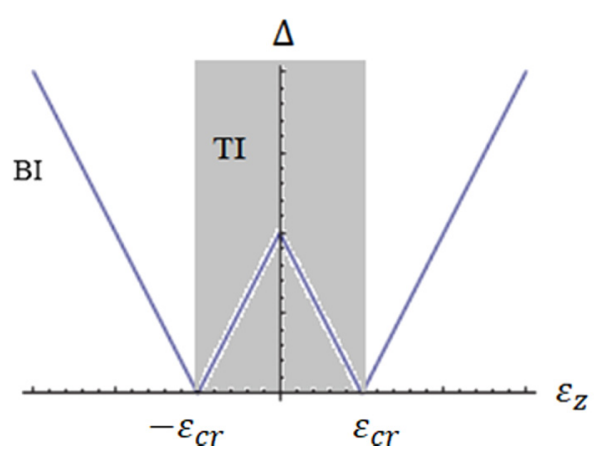

FIG. 1. Variation of band gap $\Delta$ as a function of external electric field $\varepsilon_{z}$. The gap is closed at the critical points $\varepsilon_{z}= \pm \varepsilon_{\mathrm{cr}}$. Band closing is associated with a topological phase change for Sn such that for $\left|\varepsilon_{z}\right|>\varepsilon_{\mathrm{cr}} \mathrm{Sn}$ is a band insulator (BI), while otherwise it is a topological insulator (TI). 


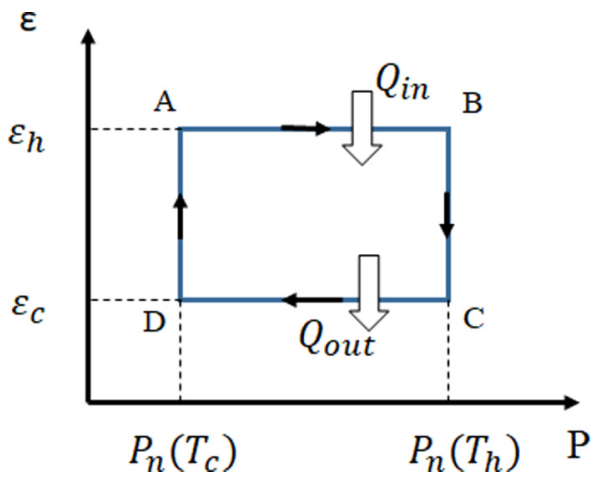

FIG. 2. Quantum Otto cycle operating between a hot bath at temperature $T_{h}$ and a cold bath at temperature $T_{c}$. It has two isochoric ( $A$ to $B$ and $C$ to $D$ ) and adiabatic ( $B$ to $C$ and $D$ to $A$ ) processes for Sn under an electric field $\varepsilon_{z}$. $Q_{\text {in }}$ is the heat injected in the $A-B$ stage, and $Q_{\text {out }}$ is the heat ejected in the $C$ - $D$ stage. The electric field changes between $\varepsilon_{h}$ and $\varepsilon_{c}$. Occupation probabilities $P$ of an energy level $E_{n}$ change between $P_{n}\left(T_{c}\right)$ and $P_{n}\left(T_{h}\right)$.

\section{RESULTS AND DISCUSSION}

\section{A. Quantum Otto cycle}

A quantum Otto cycle (QOC) consists of two isochoric and two adiabatic processes [16]. We consider a QOC with a topological insulator as a working substance. The direction of the cycle is chosen such that net positive work is produced, as is depicted in Fig. 2. The four stages of the QOC are as follows:

(i) Stage 1 ( $A$ to $B$ ): This is a quantum isochoric process where the working substance in an external electric field, $\varepsilon_{h}$, with energy levels $E_{n}^{h}$ is coupled to a hot bath at temperature $T_{h}$. At point $B$, the working substance reaches thermal equilibrium with the hot bath, and the occupation probability of each eigenstate becomes $P_{n}\left(T_{h}\right)$, while the energy levels remain the same. No work is done, but heat $Q_{\text {in }}$,

$$
Q_{\text {in }}=\int \frac{d^{2} \vec{k}}{(2 \pi)^{2}} \sum_{n} g\left(E_{n}\right) E_{n}^{h}(k)\left[P_{n}\left(T_{h}\right)-P_{n}\left(T_{c}\right)\right]
$$

is absorbed by the working substance during this process. The factor $g\left(E_{n}\right)$ gives the degeneracy of the $n$th energy level. The integral over $k=|\vec{k}|$ is from 0 to infinity. The low-energy spectrum, however, is valid up to a large $k \sim 1 / a$, where $a$ is the lattice constant of Sn. The temperatures we consider are low enough to make sure that the large $k$ values cannot contribute to the integral and hence we can use the low-energy spectrum instead of the full tight-binding solutions.

(ii) Stage 2 ( $B$ to $C$ ): In this process, which is a quantum adiabatic process, the working substance is isolated from the heat bath and the electric field changes from $\varepsilon_{h}$ to $\varepsilon_{c}$, where $\varepsilon_{c}<\varepsilon_{h}$. The energy levels change from $E_{n}^{h}$ to $E_{n}^{c}$. The occupation probabilities do not change. Work is done but no heat is transferred.

(iii) Stage 3 ( $C$ to $D$ ): The working substance is subject to a constant electric field, $\varepsilon_{c}$, and is coupled to a cold bath at temperature $T_{c}<T_{h}$. The occupation probabilities at the end of this stage are $P_{n}\left(T_{c}\right)$. Heat $Q_{\text {out }}$,

$$
Q_{\text {out }}=\int \frac{d^{2} \vec{k}}{(2 \pi)^{2}} \sum_{n} g\left(E_{n}\right) E_{n}^{c}(k)\left[P_{n}\left(T_{c}\right)-P_{n}\left(T_{h}\right)\right],
$$

is ejected from the system. In Eqs. (5) and (6), $P_{n}\left(T_{i}\right)=f_{n}\left(k, \varepsilon_{i}, T_{i}\right)$ with $i=h, c, \beta_{i}=1 / k_{\mathrm{B}} T_{i}$, where $k_{\mathrm{B}}$ is the Boltzmann constant. $f_{n}\left(k, \varepsilon_{i}, T_{i}\right)=$ $1 /\left(\exp \left\{\beta_{i}\left[E_{n}^{i}(k)-E_{F}\right]\right\}+1\right)$ is the Fermi distribution function and $E_{F}$ is the Fermi energy.

(iv) Stage 4 ( $D$ to $A$ ): The system is separated from the cold bath and undergoes another quantum adiabatic process, as the electric field is changed from $\varepsilon_{c}$ to $\varepsilon_{h}$. Energy levels change from $E_{n}^{c}$ to $E_{n}^{h}$. The occupation probabilities remain the same. There is no heat transfer, but work is done.

As the probability distribution remains invariant in the two quantum adiabatic processes, the entropy will remain invariant as well and no net heat is produced. Based on this fact, the net work produced during a QOE cycle is given by

$$
\begin{aligned}
W_{\mathrm{O}}=Q_{\text {in }}+Q_{\text {out }}= & \int \frac{d^{2} \vec{k}}{(2 \pi)^{2}} \sum_{n} g\left(E_{n}\right)\left(E_{n}^{h}(k)\right. \\
& \left.-E_{n}^{c}(k)\right)\left[P_{n}\left(T_{h}\right)-P_{n}\left(T_{c}\right)\right] .
\end{aligned}
$$

The efficiency of the heat engine is $\eta_{\mathrm{O}}=W_{\mathrm{O}} / Q_{\text {in }}$. The positive work condition requires $Q_{\text {in }}>-Q_{\text {out }}$, under which the system would operate as a heat engine.

There are a number of discussions in the literature on the thermodynamics of graphene-type materials with positive and negative energy bands. It is the common treatment to limit the evaluation of the partition function to the positive energy manifold and ignore the contribution of the negative energies that is divergent [45-48]. Alternatively, a physical argument is suggested to eliminate the negative energies from the partition function calculation by assuming doped graphene [11,49]. Here we take into account both the positive and negative energy bands on both a physically and mathematically rigorous footing for a neutral $\mathrm{Sn}$. The method is presented in the Appendixes.

We take typical values for the parameters of the $\mathrm{Sn}$ TI model [32], where $\lambda_{\text {SO }}=30 \mathrm{meV}$. We let $l \varepsilon_{h}$ and $l \varepsilon_{c}$ change in the ranges of $0-40$ and $0-150 \mathrm{meV}$ for low and high temperatures, respectively. We separately consider highand low-temperature operations of the engine. For the hightemperature case, we take the temperatures of the hot and the cold baths as $T_{h}=300 \mathrm{~K}$ and $T_{c}=150 \mathrm{~K}$, respectively, while for the low-temperature case we assume $T_{h}=40 \mathrm{~K}$ and $T_{c}=30 \mathrm{~K}$.

In Fig. 3, we show the electric potential domain of the positive work, $W_{\mathrm{O}}>0$, as the dark (blue) shaded region, while the negative work domain is indicated as the light orange shaded region. The positive work region for low temperatures plotted in Fig. 3(a) shows an X-shaped structure, which deforms as the temperature increases. The positive work region for high temperatures is shown in Fig. 3(b). It expands with increasing electric fields, except for a semicircular region terminated by the critical points of the TPT at $l \varepsilon_{\mathrm{cr}}=\lambda_{\mathrm{SO}}$. Figure $3(\mathrm{~b})$ could also be plotted for negative values of the electric fields. The result would be an inversion of the positive work regions in Figs. 3(a) and 3(b) with respect to the origin. We note that 


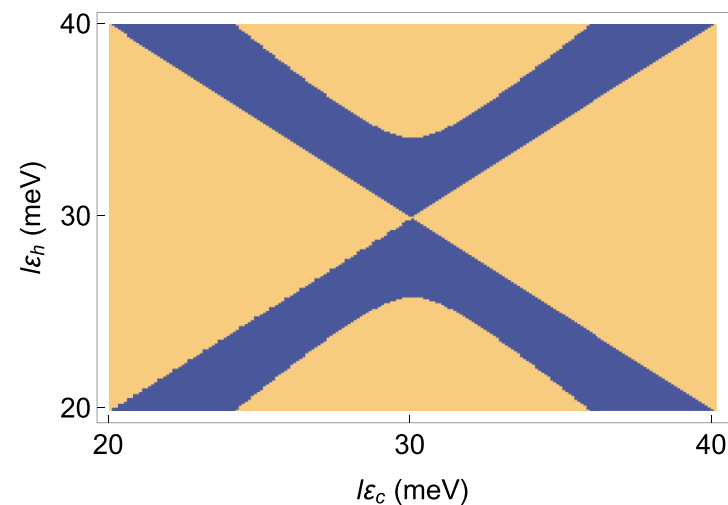

(a)

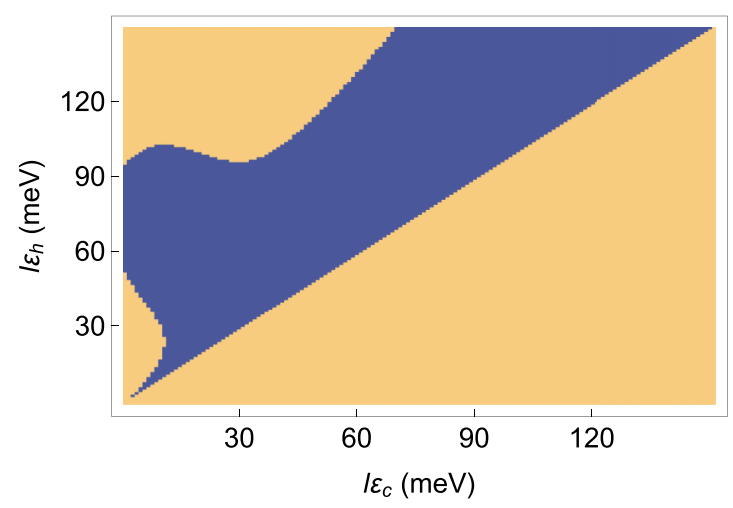

(b)

FIG. 3. Domain of positive work in the space of external electric potentials at the cold and hot isochores $l \varepsilon_{c}$ and $l \varepsilon_{h}$, respectively. Dark blue and light orange shaded regions indicate the domains of the positive and negative work. The parameters are the spin-orbit coupling $\lambda_{\mathrm{SO}}=30 \mathrm{meV}$ and the temperatures of the hot and cold baths, which are taken to be (a) $T_{h}=40 \mathrm{~K}$ and $T_{c}=30 \mathrm{~K}$ and (b) $T_{h}=300 \mathrm{~K}$ and $T_{c}=150 \mathrm{~K}$, respectively.

it is possible to extract positive work from the system even for $l \varepsilon_{h}<l \varepsilon_{c}$ for the low-temperature regime according to Fig. 3(a).

The exact values and behavior of the work output as a function of $l \varepsilon_{c}$ for three representative values of $l \varepsilon_{h}$ are plotted in Fig. 4 for low temperatures, and in Fig. 5 for high temperatures. Figure 4 shows a double-peak profile. Zeros of the work function separate the regimes of the heat engine and heat pump or refrigerator operations of the system. The number of zeros can be deduced from Fig. 3(a) by the boundary between the positive and negative work domains. The critical point of the TPT reveals itself as an extremum point in the curves at $l \varepsilon_{c}=\lambda_{\text {SO }}=30 \mathrm{meV}$. Controlling $l \varepsilon_{h}$ can change the operation of the cycle from a refrigerator to a heat engine.

For high temperatures, the same double-peak structure remains but with less symmetry between the topological and trivial insulator phases. The work value is enhanced by three orders of magnitude, as shown in Fig. 5, compared to low temperatures. It is worth noting that in the high-temperature regime, greater electric potential values are required to operate in the heat engine regime, and to observe the topological phase-transition structure.

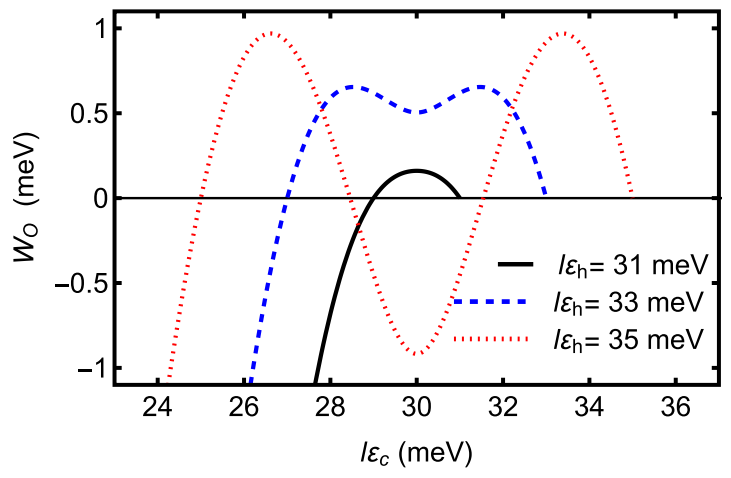

FIG. 4. Work output $W_{\mathrm{O}}$ of the quantum Otto cycle operating between the hot and cold bath temperatures $T_{h}=40 \mathrm{~K}$ and $T_{c}=$ $30 \mathrm{~K}$, respectively, as a function of the electric potential at the cold isochore $l \varepsilon_{c}$ for different values of the electric potential at the hot isochore $l \varepsilon_{h}=31 \mathrm{meV}$ (black solid), $l \varepsilon_{h}=33 \mathrm{meV}$ (blue long dashed), and $l \varepsilon_{h}=35 \mathrm{meV}$ (red short dashed). SO coupling is $\lambda_{\text {SO }}=30 \mathrm{meV}$.

The double-peak profile appears in the efficiency behavior as well, as shown in Fig. 6, where we take $l \varepsilon_{h}=35 \mathrm{meV}$ for low temperature. Qualitatively similar behaviors are found for the high-temperature case. Figure 7 indicates that the efficiency is higher for the high-temperature case, where $l \varepsilon_{h}=90 \mathrm{meV}$.

We remark that unless special procedures such as transitionless drives are used [50], the speed of the cycle would be limited by the quantum adiabatic theorem. An alternative is to remove the adiabatic stages from the cycle completely. In the following subsection, we will consider a nonadiabatic cycle, which is used in Ref. [24] to explore the signatures of a quantum phase transition.

\section{B. Stirling cycle}

It is reported in Ref. [24] that the signatures of a quantum phase transition, associated with a level crossing, can be found

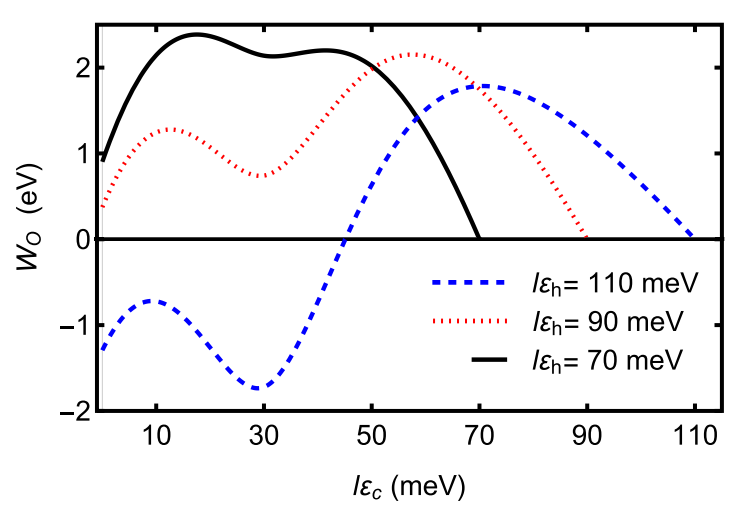

FIG. 5. Work output $W_{\mathrm{O}}$ of the quantum Otto cycle operating between the hot and cold bath temperatures $T_{h}=300 \mathrm{~K}$ and $T_{c}=$ $150 \mathrm{~K}$, respectively, as a function of the electric potential at the cold isochore $l \varepsilon_{c}$ for different values of the electric potential at the hot isochore $l \varepsilon_{h}=110 \mathrm{meV}$ (blue long dashed), $l \varepsilon_{h}=90 \mathrm{meV}$ (red short dashed), and $l \varepsilon_{h}=70 \mathrm{meV}$ (black solid). SO coupling is $\lambda_{\text {so }}=30 \mathrm{meV}$. 


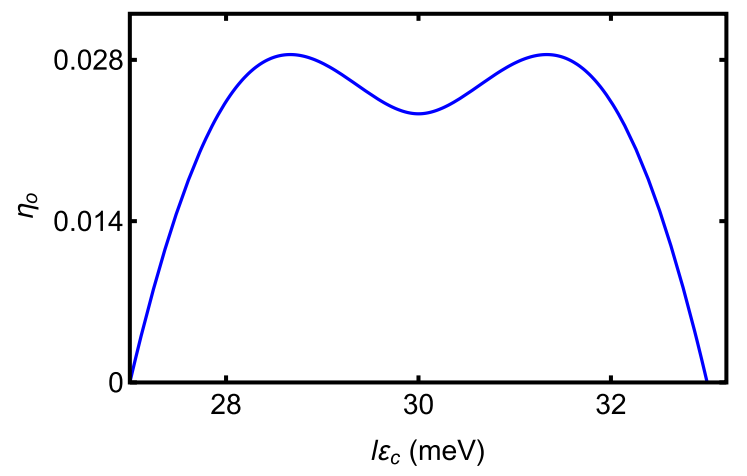

FIG. 6. Efficiency $\eta_{\mathrm{O}}$ of the quantum Otto cycle at low temperatures for the electric potential at the hot isochore $l \varepsilon_{h}=33 \mathrm{meV}$, spin-orbit coupling $\lambda_{\mathrm{SO}}=30 \mathrm{meV}$, and the temperatures of the hot and cold baths $T_{h}=40 \mathrm{~K}$ and $T_{c}=30 \mathrm{~K}$, respectively.

in the work and efficiency of a thermodynamic cycle, which consists of two isothermal and two isomagnetic processes. Isothermal processes transform the system through the phasetransition point. The TPT of Sn is associated with the band closing, and hence we may expect similar signatures of the TPT in a similar cycle considered in Ref. [24]. The cycle can be compared to that of an idealized Stirling cycle without a regenerator. We will call the cycle in Ref. [24] the Stirling cycle. In the case of $\mathrm{Sn}$, the isomagnetic stages are replaced by the isoelectric processes as shown in Fig. 8. However, the energy level structure of $\mathrm{Sn}$ is different from the model (interacting spins) in Ref. [24]. At the $K$ point, Sn can be considered as a four-level system. The only levels crossing are the two middle ones (the lowest conduction and highest valence levels).

The heat exchanged between the system and its surroundings in each stage of the cycle is given by

$$
\begin{aligned}
& Q_{B A}=T_{h}[S(B)-S(A)], Q_{C B}=U(C)-U(B), \\
& Q_{D C}=T_{c}[S(D)-S(C)], Q_{A D}=U(A)-U(D),
\end{aligned}
$$

where $U(I)$ is the internal energy at a constant electric field at each point $I=A, B, C, D$ in the cycle, and $S(I)$ is the

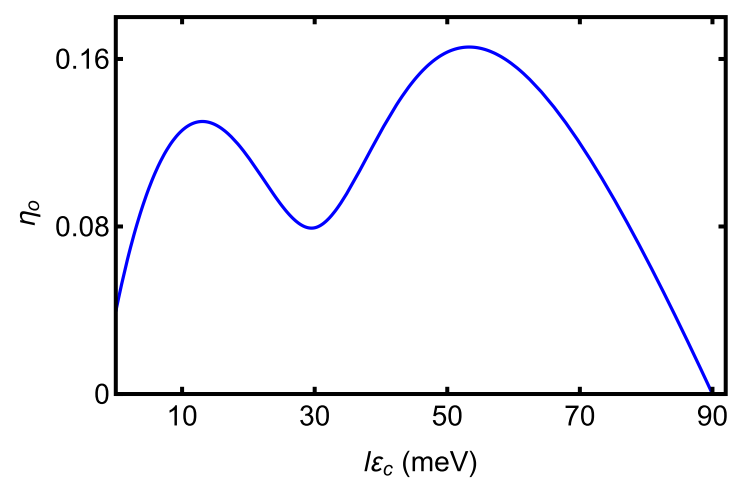

FIG. 7. Efficiency $\eta_{\mathrm{O}}$ of the quantum Otto heat engine at high temperatures for the electric potential at the hot isochore $l \varepsilon_{h}=$ $90 \mathrm{meV}$, spin-orbit coupling $\lambda_{\text {so }}=30 \mathrm{meV}$, and the temperatures of the hot and cold baths $T_{h}=300 \mathrm{~K}$ and $T_{c}=150 \mathrm{~K}$, respectively.

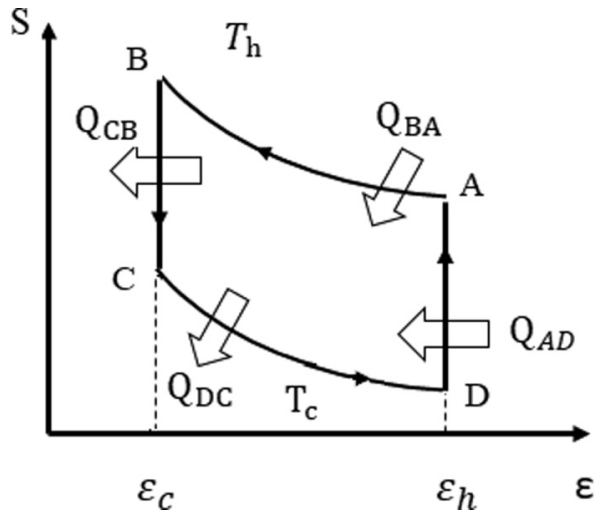

FIG. 8. Entropy-electric-field graph of a Stirling cycle consisting of two isothermal and two isoelectric stages. During the isothermal process from $A$ to $B$ ( $C$ to $D$ ), the system is brought into contact with a heat bath at temperature $T_{h}\left(T_{c}\right)$, and $Q_{B A}\left(Q_{D C}\right)$ amount of heat absorbed (released). In the isoelectric processes, the external electric field decreases (increases) from $\varepsilon_{h}\left(\varepsilon_{c}\right)$ to $\varepsilon_{c}\left(\varepsilon_{h}\right)$ and the exchanged heat is $Q_{C B}\left(Q_{A D}\right)$.

respective entropy. The total work produced in this cycle is given by

$$
W_{\mathrm{S}}=Q_{A B}+Q_{B C}+Q_{C D}+Q_{D A} .
$$

Plugging Eqs. (8) and (9), the total work is obtained in terms of the grand-canonical partition functions $\mathcal{Z}(I)$ :

$$
\begin{aligned}
W_{\mathrm{S}}= & \int \frac{d^{2} \vec{k}}{(2 \pi)^{2}}\left(\frac{1}{\beta_{h}} \ln (\mathcal{Z}(B))-\frac{1}{\beta_{h}} \ln (\mathcal{Z}(A))\right. \\
& \left.+\frac{1}{\beta_{c}} \ln (\mathcal{Z}(D))-\frac{1}{\beta_{c}} \ln (\mathcal{Z}(C))\right)
\end{aligned}
$$

where

$$
\mathcal{Z}(I)=\prod_{n}\left[1+e^{-\beta E_{n}^{i}(k)}\right]^{2} .
$$

The energy of the system $E_{n}^{i}(k)$ at point $I$ is given by Eq. (4). The definitions of internal energy and entropy and also the details of the calculations leading to Eq. (11) are given in Appendix B.

We investigate the high- and low-temperature operation of the engine separately. For both cases, we set $\lambda_{\text {so }}=30 \mathrm{meV}$ and let $\varepsilon_{c}$ change from 0 to $\varepsilon_{h}$.

In the low-temperature case, the temperatures of the hot and cold baths are $T_{h}=40 \mathrm{~K}$ and $T_{c}=30 \mathrm{~K}$, respectively. Figure 9 displays the net work and the efficiency of the cycle for the low-temperature case. In Fig. 9(a), it is observed that for different values of $l \varepsilon_{h}$ the maximum value of work occurs at $\lambda_{\text {SO }}=l \varepsilon_{c}$. These peaks identify the critical point of the TPT. Increasing the magnitude of the electric field causes a broader region of positive work. Figure 9(b), which shows the efficiency of this cycle for $l \varepsilon_{h}=40 \mathrm{meV}$, is a completely symmetric plot with a maximum at the TPT point.

For the high-temperature case, the behavior of work output as a function of $l \varepsilon_{c}$ is plotted in Fig. 10 for three different values of $T_{h}$. As the temperature of the hot bath is increased, the maximum of work is shifted away from the TPT, and the sign of the TPT point is smoothed out. 


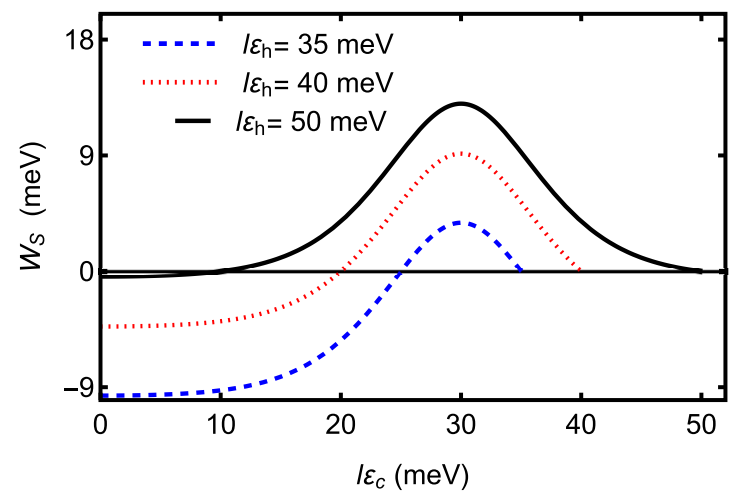

(a)

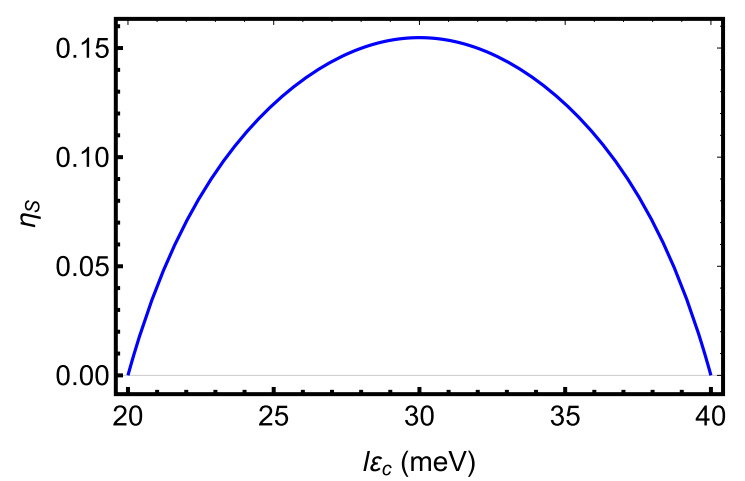

(b)

FIG. 9. (a) Work $W_{\mathrm{S}}$ of the two-level Sn model under a Stirling cycle as a function of the electric potential at the cold isoelectric stage $l \varepsilon_{c}$. The spin-orbit coupling strength is $\lambda_{\mathrm{SO}}=30 \mathrm{meV}$, hot and cold baths are at temperatures $T_{h}=40 \mathrm{~K}$ and $T_{c}=30 \mathrm{~K}$, and the electric potential at the hot isoelectric stage is $l \varepsilon_{h}=35 \mathrm{meV}$ (blue long dashed), $l \varepsilon_{h}=40 \mathrm{meV}$ (red short dashed), and $l \varepsilon_{h}=50 \mathrm{meV}$ (black solid). (b) Efficiency $\eta_{\mathrm{S}}$ of this system for $l \varepsilon_{h}=40 \mathrm{meV}$.

\section{IMPLEMENTATIONS OF THE THERMODYNAMIC CYCLES WITH TOPOLOGICAL INSULATORS}

In addition to $\mathrm{Sn}$ (or germanene or silicene) as the working substance, the basic ingredients needed to construct a roomtemperature quantum heat engine with a TPT are tunable hot and cold baths that would be periodically in contact with the monolayer TI, and a tunable electric field that will vary between two values above and below the critical point. There are experimental reports on the successful fabrication of such monolayer TIs [51-54]. The effect of an external electric field has been studied in both theoretical and experimental works $[27,36]$. While one may directly use the environment (room) temperature, more controllable environments can also be envisioned for monolayer TIs. For example, we may propose that a graphene bilayer can be used as a scaffold for Sn. Such a setup preserves the topological properties of stanene, and moreover these stacked layers are stable above room temperature [27,36]. Graphene has already been studied for its significant potential for heat flow control and energy harvesting [27,36], and there are investigations that deal with annealing graphene $[27,36]$. Accordingly, a graphene bilayer scaffold could be used to conduct heat in a controllable

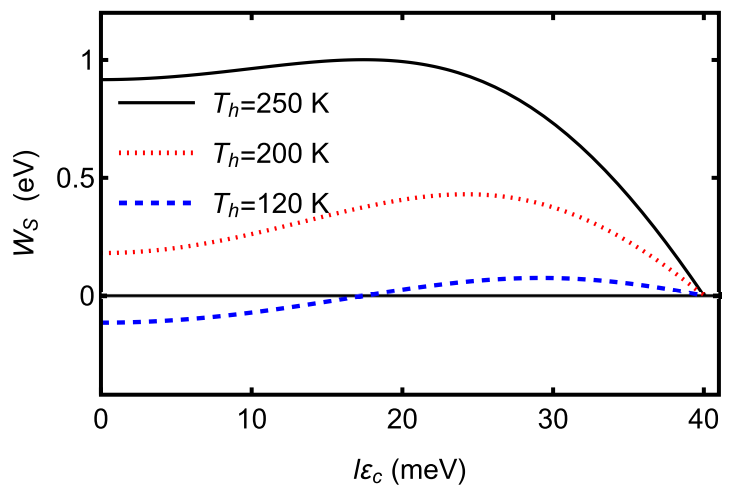

FIG. 10. Work $W_{\mathrm{S}}$ of the Stirling cycle as a function of the electric potential at the cold isochore $l \varepsilon_{c}$. The electric potential at the hot isoelectric stage is $l \varepsilon_{h}=40 \mathrm{meV}$ and the spin-orbit coupling strength is $\lambda_{\text {SO }}=30 \mathrm{meV}$. The cold bath is at temperature $T_{c}=80 \mathrm{~K}$, and the hot both is at $T_{h}=120 \mathrm{~K}$ (blue long dashed), $T_{c}=200 \mathrm{~K}$ (red short dashed), and $T_{c}=250 \mathrm{~K}$ (black solid).

manner, and it can act as a tunable hot and cold bath to implement the isothermal or isochoric heating and cooling stages of the cycles we consider. An external electric field is required for the operation of the thermodynamic cycles. This can be introduced by a capacitor.

Similar to typical experimental quantum heat engines [55] or the proposed graphene flake engine [11], our system neither stores the work nor transfers it to a work source. By including the interaction term for a work source or a load in the Sn model, such an extraction of work could be studied. In experiments, work is determined indirectly from the injected and received heat. In our case, heat can be supplied through the bottom substrate to the Sn film via electric currents with thermal noise, which could be measured to determine the work associated with the Otto and Stirling cycles.

\section{CONCLUSIONS}

We have investigated the signatures of the topological phase transition (TPT) of monolayer stanene (Sn) under an applied electric field in the work and efficiency of thermodynamic engine cycles using the $\mathrm{Sn}$ as their working substance. We considered specifically an Otto cycle and a Stirling cycle. In the case of the Otto cycle, the positive work domain in the electric-field space, as well as the magnitude of the work and efficiency, exhibit qualitative effects of the TPT. Depending on the applied fields, either a local minimum separates a doublepeaked work output profile, or the work output is maximum at the critical point of the TPT. In the Stirling cycle at low temperature, the work achieves a maximum at the TPT point, while in contrast to the Otto cycle, at high temperature, the maximum shifts away from the TPT point and the peak is smoothed out. This result generalizes the idea of Ref. [24], which uses heat engine cycles to probe ordinary quantum phase transitions (QPTs) to the probing topological QPTs. The work output is greatly enhanced as the engine operates at higher temperatures in both cycles. The system can operate either as a quantum heat engine or a refrigerator in both lowand high-temperature regimes, and the type of operation can be tuned by a wide range of applied electric fields. 
Our results are applicable to other monolayer topological insulators, such as germanene and silicene. The crucial step here is that it is established for these materials that the bulk gap closing (under electric-field variation) is associated with a topological phase transition. Our method can be an alternative to the typical scheme of probing the TPT by determining the work distribution under a sudden quench, if it is known that the bulk gap closing reflects the topological transition of the system.

The advantage of large spin-orbit coupling in such materials, relative to recently proposed graphene flake heat engines [11], is to enhance the work and efficiency with SO coupling and room- or higher-temperature operation. We hope that our results will inspire further studies of topological quantum heat engines.

\section{ACKNOWLEDGMENTS}

We acknowledge support by the Scientific and Technological Research Council of Turkey (TÜBITAK), Grant No. 117F097, and by the EU-COST Action (CA16221).

\section{APPENDIX A: WORK EXPRESSION FOR THE QUANTUM OTTO CYCLE}

The low-energy effective Hamiltonian $H_{\eta}^{s}$ for stanene has the following matrix form:

$$
\left(\begin{array}{cccc}
\eta \lambda_{\mathrm{SO}}+l \varepsilon_{z} & 0 & k_{x}+i \eta k_{y} & 0 \\
0 & -\eta \lambda_{\mathrm{SO}}+l \varepsilon_{z} & 0 & k_{x}+i \eta k_{y} \\
k_{x}-i \eta k_{y} & 0 & -\eta \lambda_{\mathrm{SO}}-l \varepsilon_{z} & 0 \\
0 & k_{x}-i \eta k_{y} & 0 & \eta \lambda_{\mathrm{SO}}-l \varepsilon_{z}
\end{array}\right),
$$

which results in the eigenenergies given by

$$
\begin{aligned}
& E^{-}(k)= \pm \sqrt{k^{2}+\left(l \varepsilon_{z}-\eta \lambda_{\mathrm{SO}}\right)^{2}}, \\
& E^{+}(k)= \pm \sqrt{k^{2}+\left(l \varepsilon_{z}+\eta \lambda_{\mathrm{SO}}\right)^{2}} .
\end{aligned}
$$

Here $\eta= \pm 1$ denotes the $K$ and $K^{\prime}$ valleys, respectively. Plugging in $\eta$, both Eqs. (A1) and (A2) result in the set of four distinct energy levels, which can be expressed in a compact form as in Eq. (4), where each energy level is twofold-degenerate. The upper two bands above the Fermi level correspond to two positive energies given in Eq. (4):

$$
\begin{aligned}
& E_{1}^{i}(k)=\sqrt{k^{2}+\left|l \varepsilon_{i}-\lambda_{\mathrm{SO}}\right|^{2}}, \\
& E_{2}^{i}(k)=\sqrt{k^{2}+\left(l \varepsilon_{i}+\lambda_{\mathrm{SO}}\right)^{2},}
\end{aligned}
$$

with $i=h, c$. We set $\hbar=v_{f}=1$. The degeneracy factor is $g\left(E_{n}^{i}\right)=2$ for each $E_{n}^{i}$, where $n=1,2$. The Fermi distribution function for neutral Sn at points $B$ and $D$ of the quantum Otto cycle is given by

$$
\begin{aligned}
& f_{n}(B)=f_{n}\left(k, \varepsilon_{h}, T_{h}\right)=\frac{1}{e^{\beta_{h} E_{n}^{h}(k)}+1}, \\
& f_{n}(D)=f_{n}\left(k, \varepsilon_{c}, T_{c}\right)=\frac{1}{e^{\beta_{c} E_{n}^{c}(k)}+1} .
\end{aligned}
$$

Due to the probability conservation on the adiabatic branches of the Otto cycle, we have $f_{n}(B)=f_{n}\left(k, \varepsilon_{h}, T_{h}\right)=$ $f_{n}(C)$ and $f_{n}(D)=f_{n}\left(k, \varepsilon_{c}, T_{c}\right)=f_{n}(A)$.
The heat received, $Q_{\text {in }}$, and ejected, $Q_{\text {out }}$, is then obtained as

$$
\begin{aligned}
Q_{\mathrm{in}}^{+} & =\int \frac{d^{2} \vec{k}}{(2 \pi)^{2}} \sum_{n=1}^{2} 2 E_{n}^{h}(k)\left[f_{n}\left(k, \varepsilon_{h}, T_{h}\right)-f_{n}\left(k, \varepsilon_{c}, T_{c}\right)\right] \\
& =\int_{0}^{\infty} d k \frac{k}{\pi}\left[E_{1}^{h}\left(f_{1}(B)-f_{1}(D)\right)+E_{2}^{h}\left(f_{2}(B)-f_{2}(D)\right)\right]
\end{aligned}
$$

and

$$
\begin{aligned}
Q_{\text {out }}^{+} & =\int \frac{d^{2} \vec{k}}{(2 \pi)^{2}} \sum_{n=1}^{2} 2 E_{n}^{c}(k)\left[f_{n}\left(k, \varepsilon_{c}, T_{c}\right)-f_{n}\left(k, \varepsilon_{h}, T_{h}\right)\right] \\
& =\int_{0}^{\infty} d k \frac{k}{\pi}\left[E_{1}^{c}\left(f_{1}(D)-f_{1}(B)\right)+E_{2}^{c}\left(f_{2}(D)-f_{2}(B)\right)\right],
\end{aligned}
$$

respectively. Superscript + of $Q$ indicates that only the positive energy bands are used in the calculation. By using the integrals given in Eqs. (A5) and (A6) in Eq. (7), the contribution of the positive energy bands to the work output can be calculated; we denote it by $W_{\mathrm{O}}^{+}$. Let us now take into consideration the negative energy bands, which can be written as

$$
\begin{aligned}
& E_{1}^{i(-)}(k)=-E_{1}^{i}(k), \\
& E_{2}^{i(-)}(k)=-E_{2}^{i}(k) .
\end{aligned}
$$

The average occupation number of these bands is given by

$$
f_{n}^{(-)}\left(k, \varepsilon_{i}, T_{i}\right)=1-f_{n}\left(k, \varepsilon_{i}, T_{i}\right) .
$$

Directly using the negative energy bands in the heat transfer calculation would lead to divergencies. We use a simple renormalization of these infinities as described below.

The ground-state energy of the system is determined by $\sum_{n=1}^{2} E_{n}^{(-)}$at $T=0$. It is not bounded from below. Nevertheless, we can formally subtract it from the internal energy of the Sn at each point in the engine cycle. Such a simple renormalization will not change the value of the net work output, as the additional terms will cancel each other in the work calculation, but it allows for finding finite energies at each cycle point. The internal energy $U(I)$ at a specific point $I$ in the cycle then becomes

$$
\begin{aligned}
U(I) & =\sum_{n=1}^{2} f_{n} E_{n}+\sum_{n=1}^{2} f_{n}^{(-)} E_{n}^{(-)}-\sum_{n=1}^{2} E_{n}^{(-)} \\
& =2 \sum_{n=1}^{2} f_{n} E_{n} .
\end{aligned}
$$

For notational simplicity, the integral over $k$, superscripts, and the arguments associated with point $I$ in $f_{n}$ and $E(k)$ are suppressed. The result in Eq. (A9) is obtained in light of Eqs. (A7) and (A8). Accordingly, the work output of the cycle can be determined by using the relation $W_{\mathrm{O}}=2 W_{\mathrm{O}}^{+}$. 


\section{APPENDIX B: WORK EXPRESSION FOR THE STIRLING CYCLE}

At each point in the Stirling cycle given in Fig. 8, we evaluate the Fermi-Dirac distribution as

$$
\begin{gathered}
f_{n}(A)=f_{n}\left(k, T_{h}, \varepsilon_{h}\right), \\
f_{n}(B)=f_{n}\left(k, T_{h}, \varepsilon_{c}\right), \\
f_{n}(C)=f_{n}\left(k, T_{c}, \varepsilon_{c}\right), \\
f_{n}(D)=f_{n}\left(k, T_{c}, \varepsilon_{h}\right) .
\end{gathered}
$$

The incoming and outgoing heat are

$$
Q_{\text {in }}=Q_{B A}+Q_{A D}=T_{h}(S(B)-S(A))+U(D)-U(A),
$$

$$
Q_{\mathrm{out}}=Q_{C B}+Q_{D C}=U(C)-U(B)+T_{c}(S(D)-S(C)) \text {. }
$$

To calculate work $W_{\mathrm{S}}=Q_{\text {in }}+Q_{\text {out }}$, we need to compute quantities like $T_{j} S(I)-U(I)$, using

$$
U^{+}(I)=2 \int \frac{d^{2} \vec{k}}{(2 \pi)^{2}} \sum_{n=1}^{2} E_{n}^{i}(k) f_{n}(I),
$$

with $E_{n}^{i}(k)$ being the corresponding eigenenergy at $I$, and

$$
\begin{aligned}
T_{j} S^{+}(I)= & -\frac{2}{\beta_{j}} \int \frac{d^{2} \vec{k}}{(2 \pi)^{2}} \sum_{n=1}^{2}\left[\left(1-f_{n}(I)\right) \ln \left(1-f_{n}(I)\right)\right. \\
& \left.+f_{n}(I) \ln \left(f_{n}(I)\right)\right] .
\end{aligned}
$$

Again the superscripts remind us that we limit ourselves to the positive energy manifold in these calculations. Equations (B7) and (B8) yield

$$
T_{j} S^{+}(I)-U^{+}(I)=\frac{2}{\beta_{j}} \int \frac{d^{2} \vec{k}}{(2 \pi)^{2}} \sum_{n=1}^{2} \ln \left(1+e^{-\beta_{j} E_{n}^{i}(k)}\right) .
$$

Using Eq. (B9) in Eq. (10), we determine the work associated with the positive energy bands and denote it as $W_{\mathrm{S}}^{+}$. The work expression for the Stirling cycle given in Eq. (11) is valid for any number of $n$.

To include the effect of the negative energy manifold, we first realize that $T_{j} S^{+}(I)=T_{j} S^{-}(I)$. Then we can employ the same method, described in Appendix A, to renormalize the divergent contribution of negative energies to the internal energy $U(I)$ by subtracting the zero-temperature ground-state energy at each point $I$ in the Stirling cycle. This leads to $U(I)=2 U^{+}(I)$. Hence we conclude that inclusion of the negative energies simply doubles the value of $T_{j} S^{+}(I)-$ $U^{+}(I)$, and accordingly we have $W_{\mathrm{S}}=2 W_{\mathrm{S}}^{+}$. We mention a subtle technical point that the ground-state energy term formally depends on the associated electric potential at the corresponding point $I$. Thus, we should designate the groundstate energy terms subtracted from the internal energies at points $A$ and $D$ of the Stirling cycle by $R\left(\varepsilon_{h}\right)$, and at points $B$ and $C$ by $R\left(\varepsilon_{c}\right)$. The heat exchanged at the isoelectric branches including these extra terms can then be expressed as

$$
\begin{aligned}
& Q_{B A}=T_{h}[S(B)-S(A)]-R\left(\varepsilon_{c}\right)+R\left(\varepsilon_{h}\right), \\
& Q_{D C}=T_{c}[S(D)-S(C)]-R\left(\varepsilon_{h}\right)+R\left(\varepsilon_{c}\right),
\end{aligned}
$$

from which we see that the extra terms cancel properly in the calculation of the net work output of the cycle by Eq. (10).
[1] D. S. L. Cardwell, From Watt to Clausius: The rise of thermodynamics in the early industrial age, Library Quart. 43, 168 (1973).

[2] H. E. D. Scovil and E. O. Schulz-DuBois, Three-Level Masers as Heat Engines, Phys. Rev. Lett. 2, 262 (1959).

[3] M. O. Scully, M. S. Zubairy, G. S. Agarwal, and H. Walther, Extracting work from a single heat bath via vanishing quantum coherence, Science 299, 862 (2003).

[4] C. A. Ryan, O. Moussa, J. Baugh, and R. Laflamme, Spin Based Heat Engine: Demonstration of Multiple Rounds of Algorithmic Cooling, Phys. Rev. Lett. 100, 140501 (2008).

[5] J. Roßnagel, S. T. Dawkins, K. N. Tolazzi, O. Abah, E. Lutz, F. Schmidt-Kaler, and K. Singer, A single-atom heat engine, Science 352, 325 (2016).

[6] A. Ü. C. Hardal and Ö. E. Müstecaplığlu, Superradiant quantum heat engine, Sci. Rep. 5, 12953 (2015).

[7] K. Zhang, F. Bariani, and P. Meystre, Quantum Optomechanical Heat Engine, Phys. Rev. Lett. 112, 150602 (2014).

[8] A. Ü. C. Hardal, N. Aslan, C. M. Wilson, and Ö. E. Müstecaplığlu, Quantum heat engine with coupled superconducting resonators, Phys. Rev. E 96, 062120 (2017).

[9] F. J. Peña, M. Ferré, P. A. Orellana, R. G. Rojas, and P. Vargas, Optimization of a relativistic quantum mechanical engine, Phys. Rev. E 94, 022109 (2016).
[10] E. Muñoz and F. J. Peña, Quantum heat engine in the relativistic limit: The case of a Dirac particle, Phys. Rev. E 86, 061108 (2012).

[11] F. J. Peña and E. Muñoz, Magnetostrain-driven quantum engine on a graphene flake, Phys. Rev. E 91, 052152 (2015).

[12] E. Muñoz, F. J. Peña, and A. González, Magnetically-driven quantum heat engines: The quasi-static limit of their efficiency, Entropy 18, 173 (2016).

[13] A. Mani and C. Benjamin, Strained-graphene-based highly efficient quantum heat engine operating at maximum power, Phys. Rev. E 96, 032118 (2017).

[14] C. V. Johnson, Holographic heat engines, Class. Quantum Grav. 31, 205002 (2014).

[15] J.-P. Brantut, C. Grenier, J. Meineke, D. Stadler, S. Krinner, C. Kollath, T. Esslinger, and A. Georges, A thermoelectric heat engine with ultracold atoms, Science 342, 713 (2013).

[16] H. T. Quan, Y.-X. Liu, C. P. Sun, and F. Nori, Quantum thermodynamic cycles and quantum heat engines, Phys. Rev. E 76, 031105 (2007).

[17] G. Benenti, G. Casati, K. Saito, and R. S. Whitney, Fundamental aspects of steady-state conversion of heat to work at the nanoscale, Phys. Rep. 694, 1 (2017).

[18] R. Alicki and R. Kosloff, Introduction to quantum thermodynamics: History and prospects, arXiv:1801.08314. 
[19] S. Vinjanampathy and J. Anders, Quantum thermodynamics, Contemp. Phys. 57, 545 (2016).

[20] J. Goold, M. Huber, A. Riera, L. del Rio, and P. Skrzypczyk, The role of quantum information in thermodynamics-A topical review, J. Phys. A 49, 143001 (2016).

[21] J. Klatzow, J. N. Becker, P. M. Ledingham, C. Weinzetl, K. T. Kaczmarek, D. J. Saunders, J. Nunn, I. A. Walmsley, R. Uzdin, and E. Poem, Experimental demonstration of quantum effects in the operation of microscopic heat engines, arXiv:1710.08716.

[22] Y. Zou, Y. Jiang, Y. Mei, X. Guo, and S. Du, Quantum Heat Engine Using Electromagnetically Induced Transparency, Phys. Rev. Lett. 119, 050602 (2017).

[23] M. Campisi and R. Fazio, The power of a critical heat engine, Nat. Commun. 7, 11895 (2016).

[24] Y.-H. Ma, S.-H. Su, and C.-P. Sun, Quantum thermodynamic cycle with quantum phase transition, Phys. Rev. E 96, 022143 (2017).

[25] E. Mascarenhas, H. Bragança, R. Dorner, M. França Santos, V. Vedral, K. Modi, and J. Goold, Work and quantum phase transitions: Quantum latency, Phys. Rev. E 89, 062103 (2014).

[26] A. Silva, Statistics of the Work Done on a Quantum Critical System by Quenching a Control Parameter, Phys. Rev. Lett. 101, 120603 (2008).

[27] S. Murakami, Gap closing and universal phase diagrams in topological insulators, Physica E 43, 748 (2011).

[28] M. Ezawa, A topological insulator and helical zero mode in silicene under an inhomogeneous electric field, New J. Phys. 14, 033003 (2012).

[29] Y. Xu, P. Tang, and S.-C. Zhang, Large-gap quantum spin Hall states in decorated stanene grown on a substrate, Phys. Rev. B 92, 081112 (2015).

[30] Y. Xu, B. Yan, H.-J. Zhang, J. Wang, G. Xu, P. Tang, W. Duan, and S.-C. Zhang, Large-Gap Quantum Spin Hall Insulators in Tin Films, Phys. Rev. Lett. 111, 136804 (2013).

[31] S. Cahangirov, M. Topsakal, E. Aktürk, H. Şahin, and S. Ciraci, Two- and One-Dimensional Honeycomb Structures of Silicon and Germanium, Phys. Rev. Lett. 102, 236804 (2009).

[32] C.-C. Liu, H. Jiang, and Y. Yao, Low-energy effective Hamiltonian involving spin-orbit coupling in silicene and twodimensional germanium and tin, Phys. Rev. B 84, 195430 (2011).

[33] M. Fadaie, N. Shahtahmassebi, M. R. Roknabad, and O. Gulseren, Investigation of new two-dimensional materials derived from stanene, Comput. Mater. Sci. 137, 208 (2017).

[34] M. Houssa, B. van den Broek, K. Iordanidou, A. K. A. Lu, G. Pourtois, J.-P. Locquet, V. Afanas'ev, and A. Stesmans, Topological to trivial insulating phase transition in stanene, Nano Res. 9, 774 (2016).

[35] M. Fadaie, N. Shahtahmassebi, and M. R. Roknabad, Effect of external electric field on the electronic structure and optical properties of stanene, Opt. Quantum Electron. 48, 440 (2016).

[36] J. E. Moore and L. Balents, Topological invariants of timereversal-invariant band structures, Phys. Rev. B 75, 121306 (2007).

[37] F.-F. Zhu, W.-J. Chen, Y. Xu, C.-L. Gao, D.-D. Guan, C.-H. Liu, D. Qian, S.-C. Zhang, and J.-F. Jia, Epitaxial growth of two-dimensional stanene, Nat. Mater. 14, 1020 (2015).
[38] Y. Xu, Z. Gan, and S.-C. Zhang, Enhanced Thermoelectric Performance and Anomalous Seebeck Effects in Topological Insulators, Phys. Rev. Lett. 112, 226801 (2014).

[39] P. Molignini, E. van Nieuwenburg, and R. Chitra, Sensing Floquet-Majorana fermions via heat transfer, Phys. Rev. B 96, 125144 (2017).

[40] A. Quelle, E. Cobanera, and C. M. Smith, Thermodynamic signatures of edge states in topological insulators, Phys. Rev. B 94, 075133 (2016).

[41] S. N. Kempkes, A. Quelle, and C. M. Smith, Universalities of thermodynamic signatures in topological phases, Sci. Rep. 6, 38530 (2016).

[42] O. Viyuela, A. Rivas, and M. A. Martin-Delgado, TwoDimensional Density-Matrix Topological Fermionic Phases: Topological Uhlmann Numbers, Phys. Rev. Lett. 113, 076408 (2014).

[43] O. Viyuela, A. Rivas, and M. A. Martin-Delgado, Uhlmann Phase as a Topological Measure for One-Dimensional Fermion Systems, Phys. Rev. Lett. 112, 130401 (2014).

[44] M. Ezawa, Monolayer topological insulators: Silicene, germanene, and stanene, J. Phys. Soc. Jpn. 84, 121003 (2015).

[45] V. Santos, R. V. Maluf, and C. A. S. Almeida, Thermodynamical properties of graphene in noncommutative phase-space, Ann. Phys. 349, 402 (2014).

[46] M. H Pacheco, R. R. Landim, and C. A. S. Almeida, Onedimensional dirac oscillator in a thermal bath, Phys. Lett. A 311, 93 (2003).

[47] A. Boumali, Thermodynamic properties of the graphene in a magnetic field via the two-dimensional Dirac oscillator, Phys. Scr. 90, 045702 (2015).

[48] M. Eshghi, H. Mehraban, and I. Ahmadi Azar, Eigenspectra and thermodynamic quantities in graphene under the inside and outside magnetic fields, Eur. Phys. J. Plus 132, 477 (2017).

[49] R. Nasir, M. A. Khan, M. Tahir, and K. Sabeeh, Thermodynamic properties of a weakly modulated graphene monolayer in a magnetic field, J. Phys.: Condens. Matter 22, 025503 (2010).

[50] M. V. Berry, Transitionless quantum driving, J. Phys. A 42, 365303 (2009).

[51] G. Zhang, H. Qin, J. Chen, X. He, L. Lu, Y. Li, and K. Wu, Growth of topological insulator $\mathrm{Bi}_{2} \mathrm{Se}_{3}$ thin films on $\mathrm{SrTiO}_{3}$ with large tunability in chemical potential, Adv. Funct. Mater. 21, 2351 (2011).

[52] C.-L. Song, Y.-L. Wang, Y.-P. Jiang, Y. Zhang, C.-Z. Chang, L. Wang, K. He, X. Chen, J.-F. Jia, Y. Wang, Z. Fang, X. Dai, X.-C. Xie, X.-L. Qi, S.-C. Zhang, Q.-K. Xue, and X. Ma, Topological insulator $\mathrm{Bi} 2 \mathrm{Se} 3$ thin films grown on double-layer graphene by molecular beam epitaxy, Appl. Phys. Lett. 97, 143118 (2010).

[53] N. Bansal, Y. S. Kim, E. Edrey, M. Brahlek, Y. Horibe, K. Iida, M. Tanimura, G.-H. Li, T. Feng, H.-D. Lee, T. Gustafsson, E. Andrei, and S. Oh, Epitaxial growth of topological insulator Bi2Se3 film on $\mathrm{Si}(111)$ with atomically sharp interface, Thin Solid Films 520, 224 (2011).

[54] X. Chen, X.-C. Ma, K. He, J.-F. Jia, and Q.-K. Xue, Molecular beam epitaxial growth of topological insulators, Adv. Mater. 23, 1162 (2011).

[55] J. Klaers, S. Faelt, A. Imamoglu, and E. Togan, Squeezed Thermal Reservoirs as a Resource for a Nanomechanical Engine Beyond the Carnot Limit, Phys. Rev. X 7, 031044 (2017). 